Commentary

\title{
Mitochondrial superoxide: a key player in Alzheimer's disease
}

\author{
Cynthia A. Massaad ${ }^{1}$, Robia G. Pautler ${ }^{1}$, and Eric Klann ${ }^{2}$ \\ ${ }^{1}$ Department of Molecular Physiology and Biophysics, Baylor College of Medicine, Houston, TX 77030, USA \\ ${ }^{2}$ Center for Neural Science, New York University, NY 10003, USA
}

\author{
Running title: Mitochondrial free radicals and Alzheimer's disease \\ Key words: Alzheimer's disease, Tg2576, SOD-2, Oxidative Stress, Mitochondria \\ Correspondence: Cynthia A. Massaad, PhD, Department of Molecular Physiology and Biophysics, Baylor College of Medicine, One \\ Baylor Plaza, BCM 335, Houston, TX 77030, USA \\ Received: 09/09/09; accepted: 09/14/09; published on line: 09/14/09 \\ E-mail: cmassaad@bcm.edu \\ Copyright: (c) 2009 Massaad et al. This is an open-access article distributed under the terms of the Creative Commons \\ Attribution License, which permits unrestricted use, distribution, and reproduction in any medium, provided the original author \\ and source are credited
}

Our recent study characterized a role for mitochondrial superoxide in the pathology of Alzheimer's disease [1]. Using the Tg2576 Alzheimer's disease (AD) mouse model in combination with a mouse that overexpresses the mitochondrial antioxidant enzyme superoxide dismutase (SOD-2), we showed that severe deficits in the spatial and associative memory of AD mice could be prevented by scavenging of superoxide. SOD-2 overexpression also resulted in a reduction in amyloid- $\beta$ $(\mathrm{A} \beta)$ plaque deposition without affecting the levels of soluble and fibrillar $A \beta$. It did however lead to a reduction in the $A \beta 42 / 40$ ratio resulting in an $A \beta$ pool composition less favorable for aggregation. These findings point towards the involvement of mitochondrial superoxide in $\mathrm{AD}$ pathology perhaps through its effects on $A \beta$ processing. Here we discuss these findings and comment on the future directions that this research may lead to.

Alzheimer's disease (AD) is a progressive neurodegenerative disease characterized by the neuropathological deposition of extracellular amyloid- $\beta(A \beta)$ plaques and intracellular tau neurofibrillary tangles [2]. The disease also is characterized by the devastating loss of memory and cognitive functions, which is thought to arise from the increased production of $A \beta$ [3]. The neurotoxic role of $\mathrm{A} \beta$ has long been established [3], however, it is not clear how it contributes to the cognitive deficits characteristic of $\mathrm{AD}$. In addition to $\mathrm{A} \beta$, several studies have implicated oxidative stress in the etiology of $\mathrm{AD}$. Oxidative stress occurs mainly as a result of overproduction of oxygen free radicals by mitochondria. The reactive oxygen species (ROS) superoxide is produced by complexes I and III of the mitochondrial respiratory chain [4], and is primarily detoxified by the mitochondrial antioxidant enzyme superoxide dismutase (SOD-2). Many lines of evidence support a pro-oxidant role for $\mathrm{A} \beta$ during $\mathrm{AD}[5,6]$. $\mathrm{A} \beta$ promotes the production of ROS in several model systems by causing dysfunction of the mitochondrial respiratory chain. Despite overwhelming evidence for the pro-oxidant role of $A \beta$, several other studies demonstrate ROS involvement prior to amyloid pathology $[7,8]$. Oxidative stress has been shown to exacerbate multiple AD phenotypes. For example, partial SOD-2 deficiency has been shown to accelerate plaque deposition [9] and increase tau phosphorylation in AD mice models [10]. SOD-2 deficiency also has been shown to accelerate the onset of a number of behavioral deficits in hAPP mice [11].

In our recent study, we investigated the involvement of mitochondrial dysfunction in $\mathrm{AD}$ pathology by studying the offspring of $\mathrm{Tg} 2576 \mathrm{AD}$ model mice that were crossed with mice that overexpress SOD-2 [1]. We found that the elevated SOD-2 reduced age-dependent increases in hippocampal superoxide, presumably from mitochondria, in the $\mathrm{Tg} 2576$ mice [1]. The reduction of hippocampal superoxide in the Tg2576 mice by SOD-2 overexpression was correlated with the prevention of spatial and associative memory deficits, as measured by the Morris water maze and fear conditioning tests, 
respectively [1]. We also measured the levels of $A \beta 40$ and $A \beta 42$ in these mice. Although we observed no difference in the absolute levels of these two peptides in the Tg2576/SOD-2 mice compared to the Tg2576 mice, we found that the ratio of $A \beta 42$ to $A \beta 40$ was reduced by the overexpression of SOD-2, which is suggestive of an $\mathrm{A} \beta$ pool less favorable for aggregation [1]. This notion was supported by histological analysis demonstrating that overexpression of SOD-2 resulted reduced amyloid plaque deposition [1].

Our data are in agreement with several studies that demonstrate the involvement of mitochondrial ROS in AD [9-12]. These studies specifically link SOD-2 deficiency with increased AD pathology and reduced mitochondrial ROS with improved cognition [9-12]. Shortly following the submission of our studies for publication, two new supporting studies were published. The first one linked $\alpha$-ketoglutarate dehydrogenase enzyme complex deficiency with increased mitochondrial ROS production and subsequent acceleration of the memory impairments and plaque deposition in Tg19959 mice [13]. The second study demonstrated mitochondrial ROS involvement in AD using a cross between the Tg19959 mouse model of AD and the SOD-2 overexpressing mice [14]. In agreement with our results, these authors demonstrated that memory impairments and increased plaque deposition in the $\operatorname{Tg} 19959$ mice were both alleviated by overexpression of SOD-2 [14]. These authors also showed that increased A $\beta 42$ levels in the $\operatorname{Tg} 19959$ mice were resistant to overexpression of SOD-2 [14]. The authors interpreted this data as evidence that the facilitation of mitochondrial antioxidant responses results in resistance to $A \beta$ and improvement of the global AD phenotype [14].

Our findings, together with the aforementioned studies, strongly suggest that mitochondrial ROS affect $A \beta$ processing. One attractive possibility is the involvement of mitochondrial ROS in the assembly of fibrillar $A \beta$ into plaques. This notion is supported by us and others, showing that quenching ROS does not affect fibrillar $\mathrm{A} \beta$ levels but reduces plaque deposition.

The involvement of oxidative stress in $\mathrm{AD}$ has long been known and investigated $[5,6,15,16]$. As a result of multiple studies linking $\mathrm{AD}$ to oxidative damage, the use of antioxidant therapy for $\mathrm{AD}$ has received considerable attention over the years. Clinical trials, however, have produced conflicting results concerning the therapeutic efficacy of antioxidant treatment for AD $[17,18]$. This may be due to either poor understanding of the pharmacokinetics or suboptimal specificity of the antioxidants compared with targeted pharmacological therapy. We and others have shown a specific involvement of mitochondrial superoxide in the etiology of $\mathrm{AD}[1,9-11,14]$. Therefore designing antioxidants targeted towards mitochondrial ROS offers an attractive therapeutic approach for AD. An important consideration to keep in mind is that the SOD-2 overexpressing mice likely have reduced levels of mitochondrial ROS beginning at birth and hence, studying the offspring generated when these mice are mated with the Tg2576 AD mice shows that quenching mitochondrial superoxide is a preventive approach to the occurrence of $\mathrm{AD}$. A more clinically relevant approach would be to determine the specific temporal window in which removal of mitochondrial ROS is most effective.

One could use either genetic or pharmacological approaches to determine whether removal of mitochondrial ROS reverses rather than prevents cognitive deficits in AD model mice. From a genetic standpoint, an attractive approach would be to study the progeny of $\mathrm{Tg} 2576 \mathrm{AD}$ mice crossed to a mouse that could be induced to express SOD-2 on demand (for example a mouse that overexpresses SOD-2 in response to tetracycline treatment - tet-on-SOD-2). Studying AD model mice that could overexpress SOD-2 at any age would permit one to determine whether diminishing mitochondrial superoxide after the onset of $\mathrm{AD}$ phenotypes could reverse symptoms of $\mathrm{AD}$, such as cognitive dysfunction and plaque deposition.

From a pharmacological standpoint, the design of antioxidants specifically targeted to mitochondria to quench mitochondrial ROS is necessary. Pharmacological catalytic scavengers of superoxide and hydrogen peroxide have proven quite effective in reversing agerelated cognitive deficits [19]. In the context of $\mathrm{AD}$, these compounds only have been tested in organotypic hippocampal cultures treated with $A \beta 42$ or $A \beta 40$ [20]. In support of our findings, $\mathrm{SOD} /$ catalase mimetics were shown to be effective against $A \beta$-induced neurotoxicity [20]. These mimetics, however, are not specific to mitochondrial ROS and do not provide information for determining the optimal temporal window in vivo for treatment of $\mathrm{AD}$. A set of synthetic plastoquinone derivatives recently were shown to specifically target the mitochondria and act as potent antioxidants at low concentrations $[21,22]$. The antioxidant properties of these derivatives (termed SKQs) have been investigated in the context of senescence and age-related impairments such as blindness [23], ischemia [24], stroke [24] and tumor development [25]. Although these agents can act as potent pro-oxidants at higher concentrations and optimization of the antioxidant therapeutic window is necessary $[21,22]$, they appear to 
be promising in terms of decelerating senescence and treating age-related diseases [21-27]. In the context of our data, which show an involvement of mitochondrial ROS in AD pathology, the use of the SKQ agents for the treatment of $\mathrm{AD}$ should be investigated.

In conclusion, our findings have reinforced the idea that mitochondrial superoxide plays a critical role in the pathological events following $\mathrm{A} \beta$ elevation during $\mathrm{AD}$. More specifically, we were able to show that increasing the expression of the mitochondrial antioxidant enzyme SOD-2 prevents memory deficits and amyloid plaque deposition associated with AD. Moreover, our findings suggest that mitochondrial superoxide may be involved in $\mathrm{A} \beta$ processing, perhaps at the level of accumulation of fibrillar $A \beta$ into plaques. The next challenge will be to determine whether quenching mitochondrial superoxide, in addition to being preventative, can be therapeutic for the treatment and reversal of cognitive dysfunction in $\mathrm{AD}$.

\section{ACKNOWLEDGEMENTS}

This work was supported by the following grants: National Institutes of Health/NINDS NS034007 and NS047384, and Alzheimer's Association Investigator Initiated Research Grant 05-15221 to EK, National Institute of Health/NIA AG029977 and The Wendler Research Fund to RGP, National Institutes of Health/NHLBI T32 HL07676 and American Health Assistance Foundation A2008-600 to CAM.

\section{CONFLICT OF INTERESTS STATEMENT}

The authors have no conflicts of interest to declare.

\section{REFERENCES}

1. Massaad CA, Washington TM, Pautler RG, Klann E. Overexpression of SOD-2 reduces hippocampal superoxide and prevents memory deficits in a mouse model of Alzheimer's disease. Proc. Natl. Acad. Sci. U.S.A. 2009;106(32):13576-13581.

2. Tanzi R, Bertram L. Twenty years of the Alzheimer's disease amyloid hypothesis: a genetic perspective. Cell. 2005;120:545555.

3. Yankner B, Dawes L, Fisher S, et al. Neurotoxicity of a fragment of the amyloid precursor associated with Alzheimer's disease. Science. 1989;245:417-420.

4. Cadenas E. Mitochondrial free radical production and cell signaling. Mol. Aspects Med. 2004;25:17-26.

5. Butterfield D, Griffin S, Munch G, Pasinetti G. Amyloid betapeptide and amyloid pathology are central to the oxidative stress and inflammatory cascades under which Alzheimer's disease brain exists. J.Alzheimers.Dis. 2002;4:193-201.

6. Behl C. Oxidative stress in Alzheimer's disease: implications for prevention and therapy. Subcell. Biochem. 2005;38:65-78.
7. Tamagno $E$, Parola $M$, Bardini $P$, et al. Beta-site APP cleaving enzyme up-regulation induced by 4-hydroxynonenal is mediated by stress-activated protein kinases pathways. J. Neurochem. 2005;92:628-636.

8. Tamagno $E$, Guglielmotto $M$, Aragno $M$, et al. Oxidative stress activates a positive feedback between the gamma- and betasecretase cleavages of the beta-amyloid precursor protein. J. Neurochem. 2008;104:683-695.

9. Li F, Calingasan N, Yu F, et al. Increased plaque burden in brains of APP mutant MnSOD heterozygous knockout mice. J. Neurochem. 2004;89:1308-1312.

10. Melov S, Adlard P, Morten K, et al. Mitochondrial oxidative stress causes hyperphosphorylation of tau. PLoS.ONE. 2007;2:e536.

11. Esposito L, Raber J, Kekonius L, et al. Reduction in mitochondrial superoxide dismutase modulates Alzheimer's disease-like pathology and accelerates the onset of behavioral changes in human amyloid precursor protein transgenic mice. J. Neurosci. 2006;26:5167-5179.

12. Du H, Guo L, Fang F, et al. Cyclophilin D deficiency attenuates mitochondrial and neuronal perturbation and ameliorates learning and memory in Alzheimer's disease. Nat. Med. 2008;14:1097-1105.

13. Dumont M, Ho DJ, Calingasan NY, et al. Mitochondrial dihydrolipoyl succinyltransferase deficiency accelerates amyloid pathology and memory deficit in a transgenic mouse model of amyloid deposition. Free Radic. Biol. Med. 2009;47:1019-1027.

14. Dumont $M$, Wille $E$, Stack $C$, et al. Reduction of oxidative stress, amyloid deposition, and memory deficit by manganese superoxide dismutase overexpression in a transgenic mouse model of Alzheimer's disease. FASEB J. 2009;23:2459-2466.

15. Zhu $X$, Lee $H$, Casadesus $G$, et al. Oxidative imbalance in Alzheimer's disease. Mol. Neurobiol. 2005;31:205-217.

16. Manczak M, Park B, Jung Y, Reddy P. Differential expression of oxidative phosphorylation genes in patients with Alzheimer's disease: implications for early mitochondrial dysfunction and oxidative damage. Neuromolecular. Med. 2004;5:147-162.

17. Engelhart $M$, Geerlings $M$, Ruitenberg $A$, et al. Dietary intake of antioxidants and risk of Alzheimer disease. JAMA. 2002;287:3223-3229.

18. Luchsinger J, Tang M, Shea S, Mayeux R. Antioxidant vitamin intake and risk of Alzheimer disease. Arch.Neurol. 2003;60:203208.

19. Liu R, Liu I, Bi X, et al. Reversal of age-related learning deficits and brain oxidative stress in mice with superoxide dismutase/catalase mimetics. Proc. Natl. Acad. Sci. U.S.A. 2003;100:8526-8531.

20. Bruce A, Malfroy B, Baudry M. beta-Amyloid toxicity in organotypic hippocampal cultures: protection by EUK-8, a synthetic catalytic free radical scavenger. Proc. Natl.Acad. ci. U.S.A. 1996;93:2312-2316.

21. Antonenko YN, Avetisyan AV, Bakeeva LE, et al. Mitochondria-targeted plastoquinone derivatives as tools to interrupt execution of the aging program. 1. Cationic plastoquinone derivatives: synthesis and in vitro studies. Biochemistry Mosc. 2008;73:1273-1287.

22. Skulachev VP, Anisimov VN, Antonenko YN, et al. An attempt to prevent senescence: a mitochondrial approach. Biochim. Biophys. Acta. 2009;1787:437-461. 
23. Neroev VV, Archipova MM, Bakeeva LE, et al. Mitochondriatargeted plastoquinone derivatives as tools to interrupt execution of the aging program. 4. Age-related eye disease. SkQ1 returns vision to blind animals. Biochemistry Mosc. 2008;73:1317-1328.

24. Bakeeva LE, Barskov IV, Egorov MV, et al. Mitochondriatargeted plastoquinone derivatives as tools to interrupt execution of the aging program. 2. Treatment of some ROS- and age-related diseases (heart arrhythmia, heart infarctions, kidney ischemia, and stroke). Biochemistry Mosc. 2008;73:1288-1299.

25. Agapova LS, Chernyak BV, Domnina LV, et al. Mitochondriatargeted plastoquinone derivatives as tools to interrupt execution of the aging program. 3. Inhibitory effect of SkQ1 on tumor development from p53-deficient cells. Biochemistry Mosc. 2008;73:1300-1316.

26. Antonenko $Y N$, Roginsky VA, Pashkovskaya $A A$, et al. Protective effects of mitochondria-targeted antioxidant SkQ in aqueous and lipid membrane environments. J. Membr. Biol. 2008;222:141-149.

27. Antonenko $\mathrm{YN}$, Avetisyan AV, Bakeeva LE, et al. Mitochondria-targeted plastoquinone derivatives as tools to interrupt execution of the aging program. 1. Cationic plastoquinone derivatives: synthesis and in vitro studies. Biochemistry Mosc. 2008;73:1273-1287. 